$54^{\text {ème }}$ Congrès de la SFMBCB, 03004 (2011)

DOI: $10.1051 / \mathrm{sfmbcb} / 20115403004$

(C) Owned by the authors, published by EDP Sciences, 2011

\title{
Que peut cacher une hémorragie buccale? A propos de deux cas cliniques
}

\author{
Mezghiche Y, Lattafi R, Saari B \\ Service de Pathologie et Chirurgie buccale, CHU Mustapha, Alger, Algérie \\ myasmin@hotmail.fr
}

En pratique odonto-stomatologique, on peut se trouver confronté à des patients présentant des troubles de l'hémostase. Si dans l'immense majorité des cas, ces troubles sont connus par le patient, il existe des situations où des manifestations odonto-stomatologiques, comme une gingivorragie, peuvent être révélatrices d'une hémopathie.

Deux cas cliniques d'hémorragie buccale révélant des affections hématologiques sont présentés, tout en insistant sur l'intérêt d'une bonne démarche diagnostique basée sur l'interrogatoire, l'examen clinique local et général, et complétée par une exploration biologique.

$1^{\text {er }}$ cas : Mme K M, âgée de 60 ans, est adressée en mars 2005 pour un détartrage à la suite d'une gingivite hémorragique. La patiente décrit un saignement buccal, surtout gingival, évoluant depuis quelque temps, ce qui la motive pour consulter un chirurgien dentiste. Elle ne signale aucun antécédent pathologique particulier. L'examen buccodentaire retrouve des pétéchies sur toute la muqueuse buccale et une ulcération hémorragique étendue sur la face interne de la joue droite. On note également une salive sanguinolente. L'examen général révèle des hématomes sur la face interne des membres supérieurs. Devant cette situation clinique, la patiente est adressée dans le Service d'Hématologie et un bilan sanguin est demandé en urgence. Le diagnostic de leucémie aiguë est posé et la patiente est hospitalisée pour une prise en charge thérapeutique.

$2^{\text {ème }}$ cas : Mme D F, âgée de 76 ans, est adressée par un confrère en février 2008 pour une lésion hémorragique sur la face interne de la joue gauche évoluant depuis trois jours. L'interrogatoire ne retrouve aucun antécédent pathologique général ou stomatologique particulier et il n'y a pas eu de prise médicamenteuse récente.

Le début de la maladie est marqué par un saignement buccal spontané, surtout le matin au réveil ce qui la motive pour consulter un chirurgien dentiste. A l'examen exobuccal, on note une ecchymose cutanée dans la région labio-mentonnière gauche avec des pétéchies, surtout sur la lèvre inférieure. Il existe également des pétéchies disséminées sur tout le corps. L'examen de la cavité buccale retrouve un érythème diffus intéressant toute la muqueuse buccale avec un hématome sur la face interne de la joue gauche; la patiente est édentée. Ce tableau clinique fait évoquer un trouble de l'hémostase et rechercher une hémopathie. Une NFS est demandée en urgence : le taux de plaquettes $3000 . \mathrm{mm}^{-3}$. La patiente est immédiatement adressée dans le Service d'Hématologie où une exploration biologique approfondie est réalisée : il s'agit d'un purpura thrombopénique.

La présentation de ces deux cas cliniques doit nous inciter à évoquer une hémopathie devant toute anomalie de l'hémostase telle qu'une gingivorragie, des pétéchies ou une ecchymose qui peuvent parfois constituer la manifestation inaugurale. En effet, les manifestations odonto-stomatologiques de nombreuses maladies générales sont fréquentes : elles peuvent être le premier signe clinique révélateur de la pathologie. Ceci permet de rappeler le rôle important du chirurgien dentiste dans le diagnostic précoce des hémopathies ce qui permet d'accélérer la prise en charge et, par conséquent, d'améliorer le pronostic.

This is an Open Access article distributed under the terms of the Creative Commons Attribution-Noncommercial License 3.0, which permits unrestricted use, distribution, and reproduction in any noncommercial medium, provided the original work is properly cited. 